\title{
Recombinant Human Stem Cell Factor
}

National Cancer Institute

\section{Source}

National Cancer Institute. Recombinant Human Stem Cell Factor. NCI Thesaurus. Code C1410

A therapeutic glycoprotein cytokine chemically identical to or similar to endogenous human stem cell factor with hematopoietic activity. Recombinant human stem cell factor (rhSCF) binds to the receptor tyrosine kinase c-kit, which may stimulate the growth of peripheral blood progenitor cells (PBPCs). This agent works synerg istically with other hematopoietic growth factors. rhSCF may promote bone marrow recovery after myeloablative therapies and procedures. 